\title{
Influence of U-shape Accelerations of Heart Rate on Very Low Frequency Band and Heart Rate Multifractality
}

\author{
Mateusz Soliński ${ }^{1}$, Jan Gierałtowski ${ }^{1}$, Jan Jacek Żebrowski ${ }^{1}$, Pawel Kuklik ${ }^{2}$ \\ (1) Faculty of Physics, Warsaw University of Technology, Warsaw, Poland \\ (2) Department of Cardiology, Electrophysiology University Medical Center Hamburg-Eppendorf, \\ Hamburg, Germany
}

\begin{abstract}
Introduction: Heart rate variability (HRV) fluctuations have a wide frequency band and are a result of multiple contributing factors. In this study, we focused on laminar, $U$-shaped accelerations of heart rate - approximately 20-40 seconds long - in the nighttime recordings.

Methods: The influence of U-shape accelerations on HRV parameters was evaluated based on night-time $34 R R$ interval time series obtained from healthy adults. We performed calculations on recordings without top $5 \%$ intervals (in terms of RR duration), without bottom 5\% intervals and on recordings with randomly shuffled $R R$ intervals. For each recording, the VLF frequency band, Shannon and Sample Entropy, standard deviation, mean, RMSSD, Multiscale Multifractal Analysis (MMA), and symbolic analysis parameters were calculated using signals with and without modifications.

Results: U-shape accelerations accounted for $5.3 \pm 3.3 \%$ of the total recordings duration. There were significant differences between the signals without modification and without the smallest $5 \%$ intervals (thus without majority of $U$-shape periods) of the values of $H F$, $L F$ and VLF frequency bands power, Shannon and Sample Entropy, standard deviation and symbolic analysis parameters $(p<0.05)$. We also observed a significant reduction of multifractality using Multiscale Multifractal Analysis (MMA) after removing the U-shape accelerations from the recording. These effects did not occur in the surrogate data with shuffled samples $(p<0.05$ was obtained only for symbolic analysis parameters), and in case of the signals with rejected the largest $5 \%$ of intervals $(p<0.05$ only for symbolic analysis parameters and Shannon entropy).

Conclusions: Characteristic U-shape accelerations in nighttime RR time series, despite a short total duration, have a significant influence on the VLF frequency band, multifractality and the complexity of the signal. The nature of these events is not clear and requires further study.
\end{abstract}

\section{Introduction}

In general, ultra- and very low frequencies in RR interval time series are often associated with a large, few hours long trends represented e.g.: circadian cycles [1]. However, apart from the large-scale periodicities, the power of VLF (and partly LF) band could be also modulated by some characteristic accelerations of RR intervals, occurring especially during sleep. Such accelerations last approximately 30 seconds and have characteristic shape similar to ' $U$ ' letter (below referred to as U-shape accelerations). Previous studies [2] have shown that adding the artificial U-shape accelerations to HRV model based on stochastic processes changes multifractal properties of the simulated signals (based on the results of Multiscale Multifractal Analysis [3]). The Hurst surfaces, after adding simulated $\mathrm{U}$-shape accelerations are more similar to the surfaces obtained from real HRV signals measured from healthy people. The observations suggest that these characteristic patterns, although being a relatively small contribution to the whole of the analyzed signal, have a significant influence on the nonlinear properties of HRV.

This preliminary study is a first, simple examination of the influence of U-shape accelerations on HRV parameters. The aim of this study was to explore the impact of these events on very low frequency component (VLF) in power spectrum and the multifractality of RR series.

\section{U-shape accelerations}

The example of a $U$-shape acceleration is presented in Figure 1. 


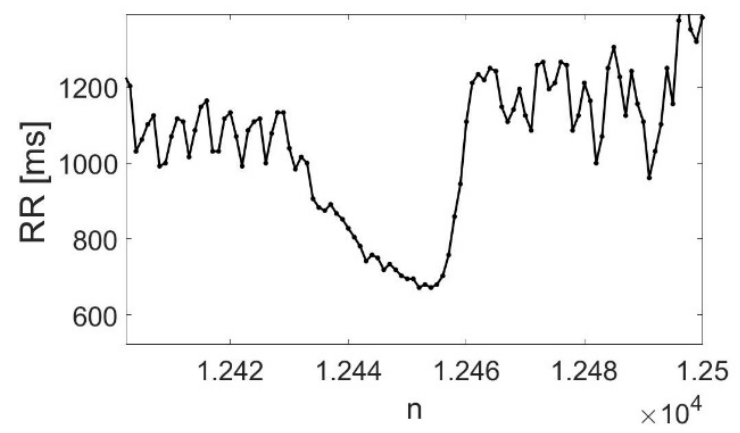

Fig. 1. An example of U-shape acceleration observed in night-time RR time interval series.

The U-shape acceleration usually occurs in a relatively smooth way until a minimum is reached. In some cases, near the minimum, a small change of the left slope is also observed: return to the mean heart rate occurs faster, i.e., the positive slope is usually steeper. Until the values of RR time intervals reach the local minimum, RR intervals decrease is usually very stable. The rise in RR intervals just after the minimum is very persistent as well.

\section{Data}

We analyzed night-time $34 \mathrm{RR}$ interval series from healthy subjects (5.6 $\pm 0.6 \mathrm{~h}$ long). All data belong to an anonymous Holter ECG database of the Institute of Cardiology (Warsaw, Poland) and were collected for medical purposes. Signals are sampled at $128 \mathrm{~Hz}$ (i.e. with a resolution of $8 \mathrm{~ms}$ ) and were checked for artifacts by a qualified cardiologist.

\section{Methods}

The influence of U-shape accelerations to HRV parameters was evaluated by analyzing RR time interval series under the following conditions:

a) $5 \%$ of the smallest $\mathrm{RR}$ intervals in the tachogram were removed,

b) $5 \%$ of the largest RR intervals in the tachogram were removed,

We assumed that all (or at least most) U-shape accelerations were removed from the signals using condition a), because of two factors:

i) in general, RR time intervals at U-shape accelerations have the lowest values in whole nighttime tachogram ii) a manual annotation of U-shape accelerations in 10 nighttime RR time series showed that the contribution of these events is around $5.3 \pm 3.3 \%$ in whole night-time signal.

The following HRV parameters were included to analysis: frequency components HFrel, LFrel and VLFrel, standard deviation (std), RMSSD, mean value, Sample Entropy, Shannon Entropy, mean h(q,s) value (MMA) and symbolic analysis parameters V0 and V2 based on Porta's method [4]. Frequency components HFrel, LFrel and VLFrel are the relative power in separate frequency bands, for example: HFrel $=($ HFrel $/($ HFrel+LFrel+VFLrel $)$. Mean $\mathrm{h}(\mathrm{q}, \mathrm{s})$ is the mean value of the Hurst exponent calculated using MMA. We compared the mean values of the HRV parameters between signals without modifications and signals under conditions a) and b) and repeated the calculations for surrogate data created by shuffling the intervals in each separate signal. We assessed the differences of the parameter values using statistical tests: t-Student or Mann-Whitney tests (depends on normality of the distribution as checked using the Kolmogorov-Smirnov test).

\section{Results}

Comparison analysis between signals without modifications and signals without 5\% of lowest values showed significant differences in the following HRV parameters $(\mathrm{p}<0.5)$ : standard deviation, HFrel, LFrel, VLFrel, Sample and Shannon entropy, mean $h(\mathrm{q}, \mathrm{s})$ exponent and symbolic analysis parameters ( $\mathrm{V} 0$ and $\mathrm{V} 2$ ). Considering the second condition (removing 5\% of upper values), we observed significant differences only for Shannon entropy and symbolic analysis parameters.

The results for surrogate data showed significant differences only for following parameters: Shannon entropy (only after removing $5 \%$ of largest values) and $\mathrm{V} 0$, V2 parameters. The mean values and standard deviations calculated for all comparisons are presented in Tables 1 and 2 .

We observed a reduction of the exponent $\mathrm{h}(\mathrm{q}, \mathrm{s})$ obtained from MMA for the signals without 5\% of lower values (where U-shape accelerations do not occur) in a wide range of scale s and fluctuations q. In contrast, the shapes of the surfaces of signals without modifications and signals 
without 5\% of upper values are very comparable. Examples of the Hurst surfaces are shown in Figure 2. In addition, there are no visible differences for surrogate data under the condition (a-b).

Table. 1. Comparison of the mean percentage differences and standard deviations of HRV parameters between signals without modifications and signals without $5 \%$ lowest or largest values. The significant differences $(p<0.05)$ were assigned as $(*)$.

\begin{tabular}{lcc}
\hline & \multicolumn{2}{c}{$\begin{array}{c}\text { Mean percentage differences } \\
\text { between signals without } \\
\text { modifications and signals: }\end{array}$} \\
\cline { 2 - 3 } HRV parameter & $\begin{array}{c}\text { without 5\% of } \\
\text { lowest values }\end{array}$ & $\begin{array}{c}\text { without } 5 \% \text { of } \\
\text { largest values }\end{array}$ \\
\hline mean & $1.23(0.27)$ & $-0.90(0.19)$ \\
std & $-18.4(5.1)^{*}$ & $-8.8(2.8)$ \\
RMSSD & $-2.2(4.2)$ & $-12.4(5.6)$ \\
HFrel & $57.6(21.0)^{*}$ & $-6.9(9.9)$ \\
LFrel & $39.2(21.3)^{*}$ & $10.2(6.1)$ \\
VLFrel & $-20.0(6.7)^{*}$ & $4.5(3.7)$ \\
Sample Entropy & $12.8(14.0)^{*}$ & $4.3(8.6)$ \\
Shannon Entropy & $1.4(1.8)^{*}$ & $2.1(0.9) *$ \\
Mean h(q,s) (MMA) & $-8.2(3.4)^{*}$ & $0.86(2.02)$ \\
V0 & $-21.9(15.4) *$ & $20.8(18.2) *$ \\
V2 & $84.6(73.4)^{*}$ & $104.8(204.8) *$ \\
\hline
\end{tabular}

Table. 2. Comparison of the mean percentage differences and standard deviations of HRV parameters between signals created by shuffling samples in each separate signal (surrogate data) and shuffled signals without $5 \%$ lowest or largest values. The significant differences $(\mathrm{p}<0.05)$ were assigned as $(*)$.

\begin{tabular}{lcc}
\hline \multirow{2}{*}{ HRV parameter } & \multicolumn{2}{c}{$\begin{array}{c}\text { Mean percentage differences } \\
\text { between signals without } \\
\text { modifications and signals: }\end{array}$} \\
\cline { 2 - 3 } & $\begin{array}{c}\text { without 5\% of } \\
\text { lowest values }\end{array}$ & $\begin{array}{c}\text { without } 5 \% \text { of } \\
\text { largest values }\end{array}$ \\
\hline mean & $1.16(0.32)$ & $-0.87(0.22)$ \\
std & $-17.5(5.7)$ & $-8.9(3.0)$ \\
RMSSD & $-17.7(6.0)$ & $-9.2(4.0)$ \\
HFrel & $-0.13(1.07)$ & $0.11(0.70)$ \\
LFrel & $0.14(1.91)$ & $0.08(1.06)$ \\
VLFrel & $0.5(2.8)$ & $-0.1(2.5)$ \\
Sample Entropy & $3.1(9.3)$ & $0.2(5.6)$ \\
Shannon Entropy & $1.4(2.0)$ & $-1.8(1.3) *$ \\
Mean h(q,s) (MMA) & $-0.5(1.1)$ & $0.4(0.7)$ \\
V0 & $-32.6(39.5) *$ & $41.6(28.7) *$ \\
V2 & $23.8(30.6) *$ & $25.4(36.8) *$ \\
\hline
\end{tabular}
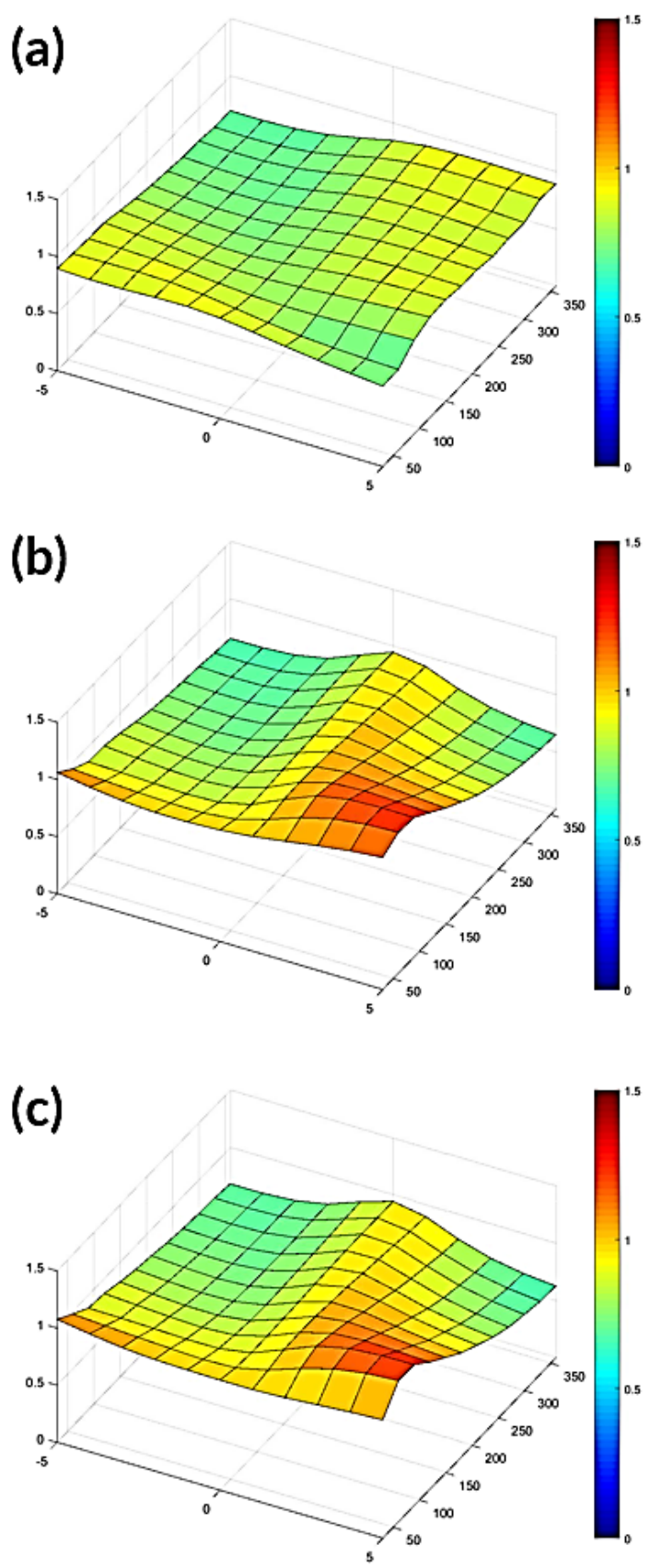

Figure 2. Example of Hurst surface obtained from MMA for tachogram under three conditions: without $5 \%$ of lowest values (a), signal without $5 \%$ of largest values (b) and signal without modifications (c). We observed a large reduction of $\mathrm{h}(\mathrm{q}, \mathrm{s})$ for a wide range of scales $\mathrm{s}$ and fluctuations $\mathrm{q}$ when the U-shape accelerations do not occur. The characteristic shape of the Hurst surface observed for a signal without modifications (c) and also without $5 \%$ of upper values (b) is destroyed. 


\section{Discussion}

The first, fundamental finding of this study is the observation of the asymmetry in the effect on HRV parameters of the removal of extreme upper and lower values (which are mainly in the form of the U-shape accelerations) from the RR time interval series. There are more significant differences between these values when the lowest values, so U-shape accelerations as well, are removed in comparison to the opposite case (removing 5\% of largest values).

The effect of U-shape accelerations on the HRV properties is also visible in parameters derived from MMA. These accelerations seem to have a large influence on persistency properties of the HRV signals which is not unexpected, since they have the most laminar and persistent structure in relation to the rest of the RR interval patterns. Moreover, they seem to increase the autocorrelation of the signal which could be directly associated with the changes in power spectrum (as results from the Wiener-Khinchin theorem [5]).

The shape of the Hurst surface after the removal of the bottom 5\% RR intervals is relatively flat and reminds rather the Hurst surface representing a noisy signal with a constant scaling exponent of around 0.8 .

The next observation is the effect of the accelerations on the VLF component. The differences in the properties of VLF between the unmodified signals and the signals with most U-shape accelerations removed suggests that this frequency component is associated with not only largescale fluctuations (e.g.: the trends connected with sleep cycles) but also relatively short, but persistent accelerations.

\section{Conclusions}

We showed an important role of the U-shape acceleration in RR time interval series - characteristic, very repetitive phenomenon occurring mainly during sleep periods.

The origin of this phenomenon is not clear. A possible explanation of $\mathrm{U}$-shape accelerations could be a reaction to increased physical activity. Another possibility is a reaction to hormonal expression, i.e. epinephrine. More detailed studies based on multimodal data (especially
EEG, hipnograms, hormonal trends and EMG signals) should be considered.

The strong effect of the removal of the smallest $5 \%$ of the values in a tachogram on HRV parameters points to a new perspective on HRV interpretation. Our results suggest that, besides the general global behavior of HRV dominated by the $1 / \mathrm{f}$ properties as shown by [6,7], it is the local, short term persistent behavior that dominates in the nonlinear properties obtained from different methods.

\section{Acknowledgements}

Mateusz Soliński acknowledges the providing medical data following people: Rafał Baranowski, Aleksander Prejbisz, Marek Kabat, Andrzej Januszewicz from The Cardinal Stefan Wyszyński Institute of Cardiology, Warsaw, Poland.

\section{References}

[1] Stein PK, Bosner MS, Kleiger RE, Conger BM, Heart rate variability: a measure of cardiac autonomic tone, American heart journal, 1994, 127(5):1376-81.

[2] Solinski M, Gieraltowski J, Żebrowski J, Modeling heart rate variability including the effect of sleep stages, Chaos, 2016, 26: 023101.

[3] Gierałtowski J, Żebrowski JJ, Baranowski R. Multiscale multifractal analysis of heart rate variability recordings with a large number of occurrences of arrhythmia, Physical Review E, 2012, 85:021915.

[4] Guzzetti S, Borroni E, Garbelli PE, Ceriani E, Della Bella P, Montano N, Cogliati C, Somers VK, Mallani A, Porta A. Symbolic dynamics of heart rate variability, Circulation, 2005, 112(4):465-70.

[5] Leibovich N, Barkai E, Aging wiener-khinchin theorem, Physical review letters, 2015, 115(8):080602.

[5] Leibovich N, Barkai E, Aging wiener-khinchin theorem, Physical review letters, 2015, 115(8):080602.

[6] Ivanov PC, Amaral LA, Goldberger AL, Havlin S, Rosenblum MG, Struzik ZR, Stanley HE, Multifractality in human heartbeat dynamics, Nature, 1999, 399:461-5.

[7] Ivanov PC, Nunes Amaral LA, Goldberger AL, Havlin S, Rosenblum MG, Stanley HE, Struzik ZR, From 1/f noise to multifractal cascades in heartbeat dynamics, Chaos: An Interdisciplinary Journal of Nonlinear Science, 2001, 11(3):641-52.

Address for correspondence.

Mateusz Soliński

Warsaw University of Technology

Faculty of Physics

Koszykowa 75, 00-662, Warsaw, Poland

solinski@if.pw.edu.pl 